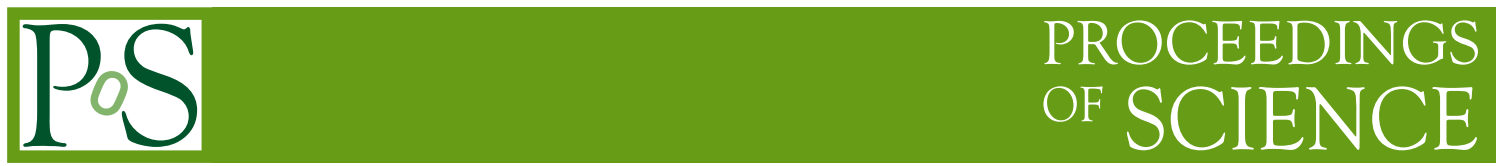

\title{
Spin Filter and GPDs in DVCS Amplitudes
}

\section{Chueng-Ryong $\mathbf{J i}^{* \dagger}$}

Department of Physics, North Carolina State University, Raleigh, NC 27695-8202, USA

E-mail: jiencsu.edu

\section{Bernard L. G. Bakker}

Department of Physics and Astrophysics, Vrije Universiteit, Amsterdam, The Netherlans

E-mail: blg.bakkerefew.vu.nl

We discuss the issue of kinematics in computing deeply virtual Compton scattering (DVCS) in terms of the widely used reduced operators that define generalized parton distributions (GPDs). Analyzing the complete tree level Compton scattering process which includes the lepton part of the amplitude, we find a dramatic difference in the result depending on whether the kinematics includes the hard transverse photon momenta or not. Our tree-level complete DVCS amplitude including the lepton current plays the role of a spin filter to analyze such kinematic dependence on the contribution of longitudinally polarized virtual photons as well as the conservation of angular momentum.

Light Cone 2010: Relativistic Hadronic and Particle Physics

June 14-18, 2010

Valencia, Spain

\footnotetext{
${ }^{*}$ Speaker.

${ }^{\dagger}$ This work is supported in part by the U.S. Department of Energy (No.DE-FG02-03ER41260).
} 


\section{Introduction}

For some time already, it has been realized that in non-forward kinematics, e.g. deeply virtual Compton scattering (DVCS), the scattering amplitudes, and thus cross sections, can be expressed in terms of objects, generalized parton distributions (GPDs), which complement the knowledge encoded in parton distribution functions $[1,2,3]$. This idea has inspired many authors, whose work has been summarized in several important review papers $[4,5,6]$.

The paramount feature of the treatment of deep inelastic scattering (DIS) and DVCS is factorization, i.e., writing the full scattering amplitude as a convolution of a hard-scattering amplitude to be calculated in perturbation theory, and a soft part embodying the hadronic structure. The use of a hard photon that is far off-shell, say $-q^{2}=Q^{2} \gg$ any relevant soft mass scale, enables factorization theorems [7] with the identification of the hard scattering amplitude. Light-front dynamics (LFD) (see e.g. Ref. [8]) can be invoked to further analyze the physics, as it has the advantage that vacuum diagrams are either rigorously absent or suppressed. In the context of single-photon physics (e.g. hadron form factors), it means that in a reference frame where the momentum of the photon $q^{\mu}$ has vanishing plus component [9]: $q^{+} \equiv\left(q^{0}+q^{3}\right) / \sqrt{ } 2=0$, it cannot create partons, as their momenta must have positive plus-components and these components are conserved in LFD. This simplification facilitates the partonic interpretation of amplitudes [10]. In two-photon physics such as DVCS, however, both photons cannot have vanishing plus components simultaneously and thus further investigation is called for to analyze the choice of a preferred kinematics in which the amplitudes are calculated and the link between the theoretical quantities, GPDs, and the cross sections can be established.

This paper is devoted to the issue of kinematics in computing the DVCS amplitude in terms of widely used reduced operators that define GPDs. We do so in the simplest possible setting, namely DVCS on a structure-less spin-1/2 particle. Although this might seem to preclude the discussion of the GPD formalism, we shall argue that important lessons can be learnt from the anlysis of this "bare bone" structure on top of which the GPDs are formulated.

In the next section, Section 2, we first report our "benchmark" calculation of the complete full DVCS amplitude which satisfies all the first principles such as gauge invariance and Lorentz invariance. In Section 3, we discuss the reduction of the full amplitude in the formulation of GPDs and compare the results between the full calculation and the reduced calculation. Conclusions follow in Section 4.

\section{Complete Amplitude with Lepton Current: Benchmark Calculation}

Before we get into the discussion of the GPD formalism, we first report our benchmark calculation of the complete full DVCS amplitude for the scattering of a massless lepton $\ell$ off a point-like fermion $f$ of mass $m$. In the final state, we find the scattered lepton $\ell^{\prime}$, the fermion $f^{\prime}$ with momentum $k^{\prime}$ and a (real) photon $\gamma^{\prime}$, viz $\ell \rightarrow \ell^{\prime}+\gamma^{*}, \gamma^{*}+f \rightarrow \gamma^{\prime}+f^{\prime}$. ('Complete' means that the amplitude includes the leptonic part and 'full' means that no approximations are made in the calculation of the hadronic amplitude.) The complete amplitude at tree level (see Fig. 1) can be written as

$$
\mathscr{M}=\sum_{h} \mathscr{L}\left(\left\{\lambda^{\prime}, \lambda\right\} h\right) \frac{1}{q^{2}} \mathscr{H}\left(\left\{s^{\prime}, s\right\}\left\{h^{\prime}, h\right\}\right),
$$



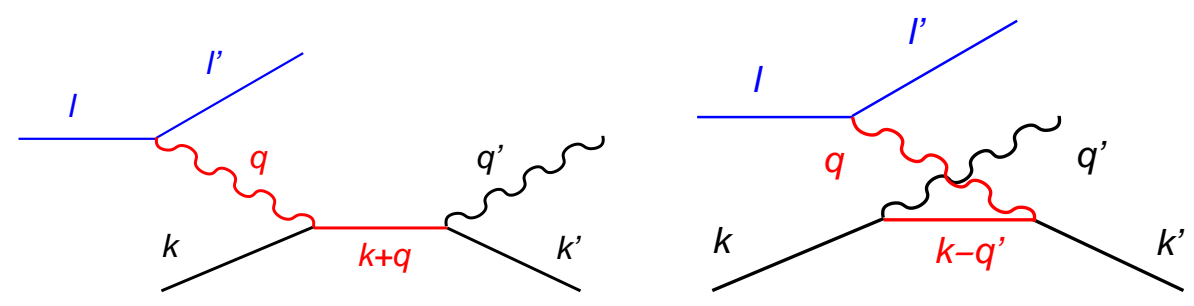

Figure 1: The two contributions to tree-level Compton scattering: $s$-channel (left), and $u$-channel (right).

where the quantities $\lambda^{\prime}, \lambda, h^{\prime}, h, s^{\prime}$, and $s$ are the helicities of the outgoing and incoming leptons, outgoing and incoming photons, and the recoiling and target fermions, respectively.

Leaving out inessential factors, we may write

$$
\mathscr{L}\left(\left\{\lambda^{\prime}, \lambda\right\} h\right)=\bar{u}\left(\ell^{\prime} ; \lambda^{\prime}\right) \phi^{*}(q ; h) u(\ell ; \lambda) ; \quad \mathscr{H}\left(\left\{s^{\prime}, s\right\}\left\{h^{\prime}, h\right\}\right)=\bar{u}\left(k^{\prime} ; s^{\prime}\right)\left(\mathscr{O}_{s}+\mathscr{O}_{u}\right) u(k ; s),
$$

where the $s$ - and $u$-channel operators of the intermediate fermion are given by

$$
\mathscr{O}_{s}=\frac{\phi^{*}\left(q^{\prime} ; h^{\prime}\right)(\not k+q+m) \phi(q ; h)}{(k+q)^{2}-m^{2}} ; \quad \mathscr{O}_{u}=\frac{\phi(q ; h)\left(\not k-q^{\prime}+m\right) \phi^{*}\left(q^{\prime} ; h^{\prime}\right)}{\left(k-q^{\prime}\right)^{2}-m^{2}} .
$$

It is straightforward to confirm that both the lepton amplitude $\mathscr{L}\left(\left\{\lambda^{\prime}, \lambda\right\} h\right)$ and the hadronic amplitude $\mathscr{H}\left(\left\{s^{\prime}, s\right\}\left\{h^{\prime}, h\right\}\right)$ satisfy the gauge invariance by substituting $\phi^{*}(q ; h)$ by $\phi$. To test the Lorentz invariance of the complete amplitude $\mathscr{M}$ given by Eq. (2.1), however, we may check if the amplitude is independent of the reference frame. In fact, in Ref. [11], we have considered three kinematics, each of which has its own merit of consideration, and confirmed that $\mathscr{M}$ is indeed identical in all three kinematics. Since the details of the kinematics and the results are presented in Ref. [11], here we just discuss the characteristic of each kinematics and the key finding from the corresponding calculation. All of the three kinematics [11], denoted as K1, K2, and K3 in the following, correspond to the hard-scattering part of a DVCS amplitude where the fermions are the quarks and $p^{+}$is the plus-component of the momentum of the parent hadron target.

\section{(K1) $\delta$-Kinematics $\left(q^{+} \rightarrow 0\right.$ as $\left.\delta \rightarrow 0\right)$}

In the $\delta \rightarrow 0$ limit, the $\delta$-kinematics coincides with the well-known $q^{+}=0$ frame [12] frequently cited in the discussion of the GPD formalism. Noticing that taking $q^{+}=0$ will lead to singular polarization vectors in the LF gauge $A^{+}=0$ (see e.g. Ref. [11]), we proceed with care: $q^{+}$is set to $\delta p^{+}$, and all amplitudes are expanded in powers of $\delta$, taking the limit $\delta \rightarrow 0$ at the very end of the calculation of the complete, physical amplitude. This replaces $q^{\mu}$ by $q_{\delta}^{\mu}$ and $q_{\delta}^{\mu}$ is given by $q_{\delta}^{\mu}=\left(\delta p^{+}, Q, 0, \frac{Q^{2}}{2(\zeta+\delta) p^{+}}+\frac{\zeta m^{2}}{2 x(x-\zeta) p^{+}}\right)$, where $x=\frac{k^{+}}{p^{+}}$is the fraction of the plus momentum of the initial quark with respect to the parent hadron target and $\zeta=\frac{q^{\prime+}-q^{+}}{p^{+}}$is the skewness in DVCS. The corresponding lepton momenta are given by $\ell^{\mu}=\left(\ell^{+}, Q, 0, \frac{Q^{2}}{2 \ell^{+}}\right)$and $\ell^{\prime \mu}=\ell^{\mu}-q_{\delta}^{\mu}$, where the value of $\ell^{+}$is determined by the on-mass-shell condition $\ell^{\prime 2}=0$ for the massless lepton.

(K2) $q^{\prime+}=0$ Kinematics (effectively, ' $1+1$ ' dim.)

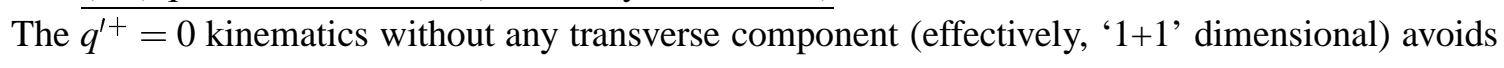


Table 1: Complete DVCS amplitudes in three kinematics, denoted as K1, K2 and K3

\begin{tabular}{|ccc|c|c|c|}
\hline & & & \multicolumn{3}{|c|}{$\sum_{h} \mathscr{L}\left(\left\{\lambda^{\prime}, \lambda\right\}, h\right) \frac{1}{q^{2}} \mathscr{H}\left(\left\{h^{\prime}, h\right\}\left\{s^{\prime}, s\right\}\right)$} \\
\hline$\left\{\lambda^{\prime}, \lambda\right\}$ & $h^{\prime}$ & $\left\{s^{\prime}, s\right\}$ & $\mathrm{K} 1$ & $\mathrm{~K} 2$ & $\mathrm{~K} 3$ \\
\hline$\left\{\frac{1}{2}, \frac{1}{2}\right\}$ & 1 & $\left\{\frac{1}{2}, \frac{1}{2}\right\}$ & $\frac{4}{Q} \sqrt{\frac{x}{x-\zeta}}$ & 0 & $\frac{4}{Q} \sqrt{\frac{x}{x-\zeta}}$ \\
$\left\{\frac{1}{2}, \frac{1}{2}\right\}$ & 1 & $\left\{-\frac{1}{2},-\frac{1}{2}\right\}$ & $\frac{4}{Q} \sqrt{\frac{x-\zeta}{x}}$ & 0 & $\frac{4}{Q} \sqrt{\frac{x-\zeta}{x}}$ \\
$\left\{-\frac{1}{2},-\frac{1}{2}\right\}$ & 1 & $\left\{\frac{1}{2}, \frac{1}{2}\right\}$ & 0 & $-\frac{4}{Q} \sqrt{\frac{x-\zeta}{x}}$ & 0 \\
$\left\{-\frac{1}{2},-\frac{1}{2}\right\}$ & 1 & $\left\{-\frac{1}{2},-\frac{1}{2}\right\}$ & 0 & $-\frac{4}{Q} \sqrt{\frac{x}{x-\zeta}}$ & 0 \\
\hline$\left\{\frac{1}{2}, \frac{1}{2}\right\}$ & -1 & $\left\{\frac{1}{2}, \frac{1}{2}\right\}$ & 0 & $\frac{4}{Q} \sqrt{\frac{x}{x-\zeta}}$ & 0 \\
$\left\{\frac{1}{2}, \frac{1}{2}\right\}$ & -1 & $\left\{-\frac{1}{2},-\frac{1}{2}\right\}$ & 0 & $\frac{4}{Q} \sqrt{\frac{x-\zeta}{x}}$ & 0 \\
$\left\{-\frac{1}{2},-\frac{1}{2}\right\}$ & -1 & $\left\{\frac{1}{2}, \frac{1}{2}\right\}$ & $-\frac{4}{Q} \sqrt{\frac{x-\zeta}{x}}$ & 0 & $-\frac{4}{Q} \sqrt{\frac{x-\zeta}{x}}$ \\
$\left\{-\frac{1}{2},-\frac{1}{2}\right\}$ & -1 & $\left\{-\frac{1}{2},-\frac{1}{2}\right\}$ & $-\frac{4}{Q} \sqrt{\frac{x}{x-\zeta}}$ & 0 & $-\frac{4}{Q} \sqrt{\frac{x}{x-\zeta}}$ \\
\hline
\end{tabular}

the singularity in the polarization vectors of the real photon and consequently provides a convenient framework of calculation without encountering any singularity. This kinematics corresponds to the special system of coordinates used by X. Ji [1] and A. Radyushkin [2] for the derivation of the GPD formalism. In the DVCS limit as $Q \rightarrow \infty$, the corresponding lepton momenta are given by $\ell^{\mu}=\left(0,0,0, \frac{Q^{2}}{2 p^{+} \zeta}\right)$ and $\ell^{\prime \mu}=\ell^{\mu}-q^{\mu}$, where $q^{\mu}=\left(-\zeta p^{+}, 0,0, \frac{Q^{2}}{2 \zeta p^{+}}\right)$.

(K3) Nonvanishing $q^{+}$and $q^{\prime+}$ Kinematics (with $m=0$ )

The nonvanishing $q^{+}$and $q^{\prime+}$ kinematics also avoids the singularity in the amplitude calculation, while the photons carry the same order of transverse momenta as the ones in the $\delta$-kinematics (K1). The corresponding lepton momenta are given by $\ell^{\mu}=\left(\ell^{+}, \frac{Q}{\sqrt{2}}, 0, \frac{Q^{2}}{4 l^{+}}\right)$and $\ell^{\prime \mu}=\ell^{\mu}-q^{\mu}$, where $q^{\mu}=\left(-\frac{\zeta}{2} p^{+}, \frac{Q}{\sqrt{2}}, 0, \frac{Q^{2}}{2 \zeta p^{+}}\right)$and the value of $\ell^{+}$is determined by $\ell^{\prime 2}=0$.

All of these three kinematics yield identical kinematical invariants such as $s=\frac{x-\zeta}{\zeta} Q^{2}$ and $u=-\frac{x}{\zeta} Q^{2}$ in the DVCS limit as $\delta \rightarrow 0$ and $m \rightarrow 0$. The results of both the lepton amplitude $\mathscr{L}\left(\left\{\lambda^{\prime}, \lambda\right\} h\right)$ and the hadronic amplitude $\mathscr{H}\left(\left\{s^{\prime}, s\right\}\left\{h^{\prime}, h\right\}\right)$ from these three kinematics can be found in Ref.[11]. The complete DVCS amplitude $\mathscr{M}$ in Eq. (2.1) is shown in Table 1. Since all the singular terms of orders $\delta^{-2}$ and $\delta^{-1}$ are exactly cancelled out in the complete amplitude, we have taken $\delta=0$ in Table 1 . Note in Table 1 that there is an interchange ${ }^{1}$ of the polarization of the final photon in the result of the ' $1+1$ ' dim. kinematics in comparison with the other kinematics, in which the momenta of photons have transverse components. This is remarkable in view of the LF helicity [13]. To appreciate this point, we draw in Fig. 2 the spin directions of the outgoing photon with the LF helicity $h^{\prime}$ for the two different kinematics: one without any transverse momentum such as K2 and the other with the transverse momentum of order $Q$ such as K1 or K3. One should

\footnotetext{
${ }^{1}$ We have also confirmed the similar interchange of the helicity amplitudes between the kinematics with and without the transverse momentum of the virtual photon in the case of a form-factor calculation.
} 


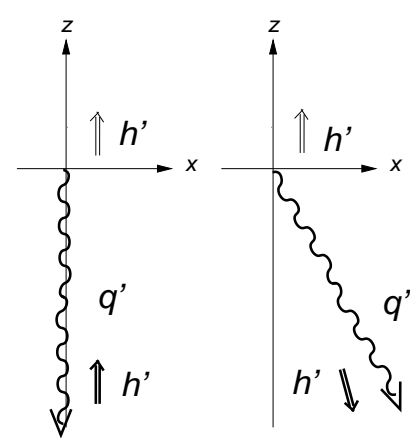

Figure 2: Spin directions corresponding to an LF boost in the $z$-direction only, 1.h.s, and one including transverse parts, r.h.s., from a state with initial spin in the $+z$-direction. Note that the spin does not align completely in the latter case.

realize that the LF helicity states are defined for a momentum $q^{\prime}$ by taking a state at rest with the spin projection along the $z$ direction equal to the desired helicity, then boosting in the $z$ direction to get the desired $q^{\prime+}$, and then doing a LF transverse boost (i.e., $E_{1}=K_{1}+J_{2}$ [13]) to get the desired transverse momentum $\vec{q}_{\perp}^{\prime}$. Whether the kinematics includes the LF transverse boost $\left(E_{1}\right)$ or not makes a dramatic difference in the spin direction because $E_{1}$ rotates the spin direction. Thus, for the 1. h. panel of Fig. 2, the spin direction of the LF helicity state is opposite (or antiparallel) to the direction of the photon momentum while for the $r$. h. panel of Fig. 2, the spin directions of the LF helicity state and the Jacob-Wick helicity state [14] are related [13] by the Wigner function $d_{h^{\prime}, h^{\prime}}^{1}\left(\tan ^{-1} \frac{2 m}{Q}\right)$ in the DVCS limit, which becomes unity as $Q \rightarrow \infty$. This illustrates the correspondence between the results of a kinematics with $\vec{q}_{\perp}^{\prime}=0$ and a kinematics with the transverse momentum of order $Q$ : e.g. in Table 1 , the result of $h^{\prime}=1$ in K2 corresponds to the result of $h^{\prime}=-1$ in $\mathrm{K} 1$ or $\mathrm{K} 3$ for $\lambda^{\prime}, \lambda=\frac{1}{2}, \frac{1}{2}$ and $s^{\prime}, s=\frac{1}{2}, \frac{1}{2}$. One should note that the conservation of angular momentum is satisfied in the complete full amplitudes for any kinematics. Thus, the calculation up to now plays the role of a benchmark for the discussion of the GPD formalism as we do below.

\section{Reduction of DVCS Amplitudes with GPDs}

Rewriting the $s$ - and $u$ - channel hadronic amplitudes (see Eqs. (2.2)-(2.3)) as

$$
\bar{u}\left(k^{\prime} ; s^{\prime}\right) \mathscr{O}_{s} u(k ; s)=\varepsilon_{\mu}{ }^{*}\left(q^{\prime} ; h^{\prime}\right) \varepsilon_{v}(q ; h) T_{s}{ }^{\mu v} ; \quad \bar{u}\left(k^{\prime} ; s^{\prime}\right) \mathscr{O}_{u} u(k ; s)=\varepsilon_{\mu}{ }^{*}\left(q^{\prime} ; h^{\prime}\right) \varepsilon_{v}(q ; h) T_{u}{ }^{\mu v},
$$

we may neglect an inessential fermion mass $m$ to express the tensorial amplitudes $T_{s}{ }^{\mu v}$ and $T_{u}{ }^{\mu v}$ as

$$
T_{s}^{\mu v}=\frac{k_{\alpha}+q_{\alpha}}{s} \bar{u}\left(k^{\prime} ; s^{\prime}\right) \gamma^{\mu} \gamma^{\alpha} \gamma^{v} u(k ; s) ; \quad T_{u}^{\mu v}=\frac{k_{\alpha}-q_{\alpha}^{\prime}}{u} \bar{u}\left(k^{\prime} ; s^{\prime}\right) \gamma^{v} \gamma^{\alpha} \gamma^{\mu} u(k ; s),
$$

respectively. Using the identity

$$
\gamma^{\mu} \gamma^{\alpha} \gamma^{v}=g^{\mu \alpha} \gamma^{v}+g^{\alpha v} \gamma^{\mu}-g^{\mu v} \gamma^{\alpha}+i \varepsilon^{\mu \alpha v \beta} \gamma_{\beta} \gamma_{5}
$$

and the Sudakov variables $n^{\mu}(+)=(1,0,0,0)$ and $n^{\mu}(-)=(0,0,0,1)$, one may expand $T_{s}{ }^{\mu v}$ and $T_{u}{ }^{\mu v}$ to find the terms proportional to $\bar{u}\left(k^{\prime} ; s^{\prime}\right) \not h(-) u(k ; s)$ and $\bar{u}\left(k^{\prime} ; s^{\prime}\right) \not h(-) \gamma_{5} u(k ; s)$ that correspond 
to the nucleon GPDs $H\left(x, \Delta^{2}, \zeta\right)$ and $\bar{H}\left(x, \Delta^{2}, \zeta\right)$ defined e.g. in Ref. [1], respectively (here, $\Delta^{2}=$ $\left.\left(q^{\prime}-q\right)^{2}\right)$. As we mentioned in Section 2 regarding K2, one should note that a special system of coordinates without involving any large transverse momentum was chosen in Refs. [1] and [2] to compute the scattering amplitude in terms of GPDs.

In order to cover the more general kinematics involving large transverse momenta such as $\mathrm{K} 1$ and K3, we may expand $q^{\mu}$ (similarly $q^{\prime \mu}$ ) and $k^{\mu}$ as $q^{\mu}=q^{+} n^{\mu}(+)+q^{-} n^{\mu}(-)+q_{\perp}{ }^{\mu}$ and $k^{\mu}=k^{+} n^{\mu}(+)+k^{-} n^{\mu}(-)$ with $q_{\perp}{ }^{\mu}$ representing the transverse momentum corresponding to $q^{\mu}$. For $m=0, k^{-}=0$, and $T_{s}{ }^{\mu v}$ (similarly $T_{u}{ }^{\mu v}$ ) can be expanded as

$$
\begin{aligned}
T_{s}^{\mu v}=\frac{1}{s}[ & \left(\left\{\left(k^{+}+q^{+}\right) n^{\mu}(+)+q^{-} n^{\mu}(-)+q_{\perp}{ }^{\mu}\right\} n^{v}(+)\right. \\
& \left.+\left\{\left(k^{+}+q^{+}\right) n^{v}(+)+q^{-} n^{v}(-)+q_{\perp}{ }^{v}\right\} n^{\mu}(+)-g^{\mu v} q^{-}\right) \bar{u}\left(k^{\prime} ; s^{\prime}\right) \not(-) u(k ; s) \\
& \left.-i \varepsilon^{\mu v \alpha \beta}\left\{\left(k^{+}+q^{+}\right) n_{\alpha}(+)+q^{-} n_{\alpha}(-)+q_{\perp \alpha}\right\} n_{\beta}(+) \bar{u}\left(k^{\prime} ; s^{\prime}\right) \not h(-) \gamma_{5} u(k ; s)\right] .
\end{aligned}
$$

Since $q^{-}$has the highest power of $Q$ among the components of momenta, one may just take the terms proportional to $q^{-}$as shown in Refs. [1] and [2], i.e.,

$$
\begin{aligned}
{T_{s}}^{\mu v}= & \frac{q^{-}}{s}\left[\left\{n^{\mu}(-) n^{v}(+)+n^{v}(-) n^{\mu}(+)-g^{\mu v}\right\} \bar{u}\left(k^{\prime} ; s^{\prime}\right) \not h(-) u(k ; s)\right. \\
& \left.-i \varepsilon^{\mu v \alpha \beta} n_{\alpha}(-) n_{\beta}(+) \bar{u}\left(k^{\prime} ; s^{\prime}\right) \not h(-) \gamma_{5} u(k ; s)\right] .
\end{aligned}
$$

Although this is correct in the frame of reference chosen in Refs. [1] and [2], one should note that Eq. (3.5) cannot provide the full result of the hadronic amplitude in the kinematics involving large transverse momenta such as $\mathrm{K} 1$ and $\mathrm{K} 3$, because the polarization vectors $\varepsilon_{\mu}{ }^{*}\left(q^{\prime} ; h^{\prime}\right)$ and $\varepsilon_{v}(q ; h)$ in Eq. (3.1) amplify the contributions neglected in the tensorial amplitude $T_{s}{ }^{\mu v}$ given by Eq. (3.5) (and similarlty for $\left.T_{u}{ }^{\mu v}\right)$. For example, the coefficient of $\bar{u}\left(k^{\prime} ; s^{\prime}\right) \not h(-) u(k ; s)$ in the $s$-channel hadronic amplitude $\bar{u}\left(k^{\prime} ; s^{\prime}\right) \mathscr{O}_{s} u(k ; s)$ is given by the following four terms:

$$
\begin{aligned}
\frac{1}{s} & {\left[2\left(k^{+}+q^{+}\right) \varepsilon^{*-}\left(q^{\prime} ; h^{\prime}\right) \varepsilon^{-}(q ; h)+\varepsilon^{*-}\left(q^{\prime} ; h^{\prime}\right) q_{\perp} \cdot \varepsilon_{\perp}(q ; h)\right.} \\
& \left.+\varepsilon^{-}(q ; h) q_{\perp} \cdot \varepsilon_{\perp}{ }^{*}\left(q^{\prime} ; h^{\prime}\right)-q^{-} \varepsilon_{\perp}^{*}\left(q^{\prime} ; h^{\prime}\right) \cdot \varepsilon_{\perp}(q ; h)\right] .
\end{aligned}
$$

Since all of the above four terms have the same powers of $Q$, one cannot just take the last term proportional to $q^{-}$but must keep all terms together. In other words, the factorization in the tensorial amplitude $T_{s}{ }^{\mu v}+T_{u}{ }^{\mu v}$ cannot hold in general because the polarization vectors $\varepsilon_{\mu}{ }^{*}\left(q^{\prime} ; h^{\prime}\right)$ and $\varepsilon_{v}(q ; h)$ can amplify the terms neglected in the tensorial amplitude unless a special system of coordinates is chosen to avoid the large transverse momenta of initial and final photons such as K2. Thus, we note that the formulation of GPDs on the level of the tensorial amplitude is not general enough to cover the kinematics with large transverse momenta such as K1 and K3 but is limited to the special system of coordinates without involving large transverse momenta such as K2.

To demonstrate the consequence of taking the reduced amplitude that keeps only the terms proportional to $q^{-}$in the tensorial amplitude as done in the formulation of GPDs, we may take the following reduced hadronic operators used in the formulation of GPDs which are defined as the limits $Q \rightarrow \infty$ of the operators given in Eq. (2.3):

$$
\left.\mathscr{O}_{s}\right|_{\operatorname{Red}}=\frac{\xi^{*}\left(q^{\prime} ; h^{\prime}\right) \gamma^{+} \phi(q ; h)}{2 p^{+}} \frac{1}{x-\zeta} ;\left.\quad \mathscr{O}_{u}\right|_{\operatorname{Red}}=\frac{\notin(q ; h) \gamma^{+} \phi^{*}\left(q^{\prime} ; h^{\prime}\right)}{2 p^{+}} \frac{1}{x} .
$$




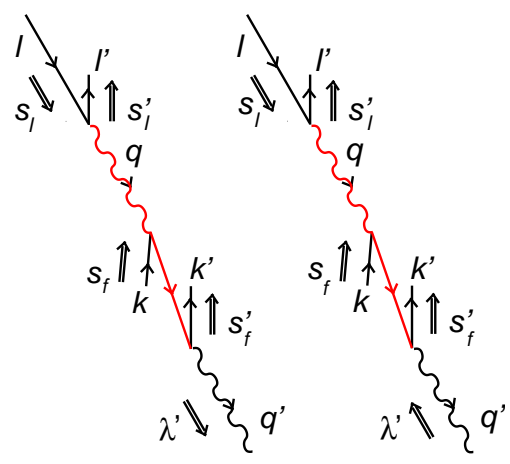

Figure 3: Due to the conservation of angular momentum, the left and right diagrams in $s$-channel are allowed and prohibited, respectively.

The $J=0$ fixed pole contribution in Eq. (3.7) for point-like scattering has been discussed in Ref. [15] along with the universality of this contribution in two-photon processes.

Since the $q^{+}=0$ frame is used [12] in the GPD formalism, we utilize the $\delta$-kinematics (K1) for our demonstration [11]. In the expansion of the hard momentum scale $Q$, it is important to retain terms of orders $\delta^{-1}, \ldots \delta^{2}$ as well as orders $Q^{-1}, \ldots Q^{2}$, as it turns out that not only are the order $\delta^{-1}$-terms cancelled by order $\delta$ terms in the convolution of $\mathscr{L}$ and $\mathscr{H}$, but also that the order $Q^{-1}$-contribution of the longitudinally polarized virtual photon gives a finite contribution in leading order. Thus, if the contribution of the longitudinal polarization of the virtual photon is neglected, the singular parts do not cancel out. Contrary to the statement in Ref. [12], the contribution of the longitudinal part is not suppressed by a factor $1 / Q$ compared to the contributions of the transversely polarized photons. Consequently, the contribution of the longitudinal polarization should not be neglected in the kinematics $\mathrm{K} 1$ and $\mathrm{K} 3$, where the photons carry transverse momenta of order $Q$.

Moreover, one can easily check the angular momentum conservation for the DVCS amplitude in the $q^{+}=0$ frame (K1) as shown in Fig. 3. From Fig. 3, one can easily see that the ampltude of $s_{l}=s_{l}^{\prime}=1 / 2, s_{f}=s_{f}^{\prime}=1 / 2$ and $\lambda^{\prime}=+1\left(\lambda^{\prime}=-1\right)$ is allowed (prohibited). Indeed, as we discussed in Section 2, our benchmark results of the complete full amplitude shown in Table 1 for all three kinematics satisfy the conservation of angular momentum. However, this is not the case for the reduced amplitudes in the GPD formalism. As we have shown in Ref. [11], the reduced amplitudes and the full ones disagree in $\mathrm{K} 1\left(q^{+}=0\right.$ frame $)$ as well as in $\mathrm{K} 3$, although for the kinematics without any transverse component, $\mathrm{K} 2$, the reduced amplitudes and the full ones do agree.

Thus, for a correct analysis of the experimental data in DVCS, one must limit the kinematics of GPDs to the reference frame where the transverse momentum of the virtual photon is not of order $Q$ but small or zero, e.g. to the center-of-mass of virtual photon and target hadron, or to the kinematics K2, as the bulk of the GPD discussion $[16,17,18]$ refers to. Since the operator that defines the GPDs, e.g. $\gamma^{+}$, is not invariant under the transformation from the $\vec{q}_{\perp}=0$ frame to the $q^{+}=0$ frame, the choice of reference frame matters in computing the DVCS amplitude in terms of GPDs. 


\section{Conclusions}

Based on our tree-level calculations of DVCS amplitudes, we find that the formulation of GPDs on the level of tensorial amplitude $T_{s}{ }^{\mu v}+T_{u}{ }^{\mu v}$ is not general enough to cover the kinematics with large transverse momenta such as $\mathrm{K} 1$ and $\mathrm{K} 3$, but is limited to the special system of coordinates without involving large transverse momenta such as $\mathrm{K} 2$. In $\mathrm{K} 1$ and $\mathrm{K} 3$, the full hadronic amplitudes and the reduced ones do not agree, even in the limit $Q \rightarrow \infty$, which means that the calculations of the DVCS amplitudes using the GPD cannot be trusted in this kinematics. In addition, the contribution of the longitudinally polarized virtual photon is not down by one order in $Q$, but even plays the role of cancelling the singular parts. The singularities we have found are in no way connected to the strong-interaction part, but entirely due to the minus components of the photon-polarization vectors, meaning that a calculation beyond tree level will encounter the same singularities.

\section{References}

[1] X. Ji, Phys. Rev. Lett. 78, 610 (1997)

[2] A.V. Radyushkin, Phys. Rev. D 56, 5524 (1997)

[3] D. Mueller, D. Robaschik, B. Geyer, F. M. Dittes and J. Horejsi, Fortsch. Phys. 42, 101 (1994)

[4] M. Diehl, Phys. Reports 388, 41 (2003)

[5] A.V. Belitsky and A.V. Radyushkin, Phys. Reports 418, 1 (2005)

[6] S. Boffi and B. Pasquini, Riv. Nuovo Cim. 30, 387 (2007)

[7] J.C. Collins, L. Frankfurt, and M. Strikman, Phys. Rev. D 56, 2982 (1997)

[8] S.J. Brodsky, H.-C. Pauli, and S.S. Pinsky, Phys. Reports 301, 299 (1998)

[9] S.D. Drell and T.M. Yan, Phys. Rev. Lett. 24, 181 (1970); G. West, ibid.24, 1206 (1970).

[10] S.J. Brodsky and S.D. Drell, Phys. Rev. D 22, 2236 (1980).

[11] B.L.G. Bakker and C.-R. Ji, arXiv:1002.0443 [hep-ph].

[12] S.J. Brodsky, M. Diehl, and D.-S. Hwang, Nucl. Phys, B 596, 99 (2001)

[13] C. Carlson and C.-R. Ji, Phys. Rev. D 67, 116002 (2003).

[14] M. Jacob and G.C. Wick, Ann. Phys. (N.Y.) 7, 404 (1959) [Ann. Phys. (N.Y.) 281, 774 (2000)]; G.C. Wick, Ann. Phys. (N.Y.)

[15] S.J. Brodsky, F.J. Llanes-Estrada, and A.P. Szczepaniak, Phys. Rev. D 79, 033012 (2009).

[16] A. V. Belitsky, D. Mueller and A. Kirchner, Nucl. Phys. B 629, 323 (2002); A. V. Belitsky and D. Mueller, Phys. Rev. D 79, 014017 (2009)

[17] J. C. Collins and A. Freund, Phys. Rev. D 59, 074009 (1999)

[18] M. Diehl, T. Gousset, B. Pire and J. P. Ralston, Phys. Lett. B 411, 193 (1997) 